\title{
Article
}

\section{On oscillatory second-order nonlinear delay differential equations of neutral type}

\section{Sandra Pinelas ${ }^{1, *}$ and Shyam Sundar Santra ${ }^{2,3}$}

1 Academia Militar, Departamento de Ciencias Exactas e Naturais, Av. Conde Castro Guimaraes, 2720-113, Amadora, Portugal.

2 Department of Mathematics, Sambalpur University, Sambalpur 768019, India.; shyam01.math@gmail.com

3 Department of Mathematics, JIS College of Engineering, Kalyani 741235, India.

* Correspondence: sandra.pinelas@gmail.com

Received: 10 July 2019; Accepted: 29 September 2019; Published: 30 November 2019.

Abstract: In this paper, new sufficient conditions are obtained for oscillation of second-order neutral delay differential equations of the form $\frac{d}{d t}\left[r(t) \frac{d}{d t}[x(t)+p(t) x(t-\tau)]\right]+q(t) G\left(x\left(t-\sigma_{1}\right)\right)+v(t) H\left(x\left(t-\sigma_{2}\right)\right)=$ $0, \quad t \geq t_{0}$, under the assumptions $\int_{0}^{\infty} \frac{d \eta}{r(\eta)}=\infty$ and $\int_{0}^{\infty} \frac{d \eta}{r(\eta)}<\infty$ for $|p(t)|<+\infty$. Two illustrative examples are included.

Keywords: Oscillation, nonoscillation, nonlinear, delay argument, second-order neutral differential equation.

MSC: 34C10, 34C15, 34K11.

\section{Introduction}

$\mathbf{T}$

his article is concerned with sufficient conditions for oscillation of a nonlinear neutral second-order delay differential equation

$$
\frac{d}{d t}\left[r(t) \frac{d}{d t} z(t)\right]+q(t) G\left(x\left(t-\sigma_{1}\right)\right)+v(t) H\left(x\left(t-\sigma_{2}\right)\right)=0, \quad t \geq t_{0},
$$

where $z(t)=x(t)+p(t) x(t-\tau)$ and $p \in P C\left(\left[t_{0}, \infty\right), \mathbb{R}\right)$. We also suppose that the following assumptions hold:

$\left(A_{1}\right) r, q, v \in C\left(\left[t_{0}, \infty\right),[0, \infty)\right), \tau, \sigma_{1}, \sigma_{2} \in \mathbb{R}_{+}$and $\rho=\max \left\{\tau, \sigma_{1}, \sigma_{2}\right\} ;$

$\left(A_{2}\right) G, H \in C(\mathbb{R}, \mathbb{R})$ with $u H(u)>0$ and $y G(y)>0$ for $u, y \neq 0$;

$\left(A_{3}\right) \int_{0}^{\infty} \frac{d \eta}{r(\eta)}=\infty$;

$\left(A_{4}\right) \int_{0}^{\infty} \frac{d \eta}{r(\eta)}<\infty$.

Baculikova et al. [1] have considered the second order delay differential equation of the form

$$
\frac{d}{d t}\left[r(t) \frac{d}{d t}[x(t)+p(t) x(\tau(t))]\right]+q(t) x(\sigma(t))+v(t) x(\eta(t))=0,
$$

where $r(t), q(t), v(t) \in C\left(\left[t_{0}, \infty\right)\right), r(t), p(t), \tau(t), \sigma(t), \eta(t) \in C^{1}\left(\left[t_{0}, \infty\right)\right)$ and established several sufficient conditions for oscillation of solution of (2) for $0 \leq p(t)<\infty$. Li et al. [2] obtained sufficient conditions for oscillation of solution of second order nonlinear neutral differential equations of the form

$$
\frac{d}{d t}\left[r(t)\left[\frac{d}{d t}[x(t)+p(t) x(t-\tau)]\right]^{\gamma}\right]+q(t) f(x(t), x(\sigma(t)))=0,
$$

where $p, q, r \in C\left(\left[t_{0},+\infty\right),(0,+\infty)\right)$ and $\gamma \geq 1$ is the quotient of two odd positive integers. In [3], Santra has consider first-order nonlinear neutral delay differential equations of the form

$$
\frac{d}{d t}[x(t)+p(t) x(t-\tau)]+q(t) H(x(t-\sigma))=f(t)
$$


and

$$
\frac{d}{d t}[x(t)+p(t) x(t-\tau)]+q(t) H(x(t-\sigma))=0
$$

and studied oscillatory behaviour of the solutions of Equation (3) and Equation (4), under various ranges of $p(t)$. Also, sufficient conditions are obtained for existence of bounded positive solutions of (3). Tripathy et al. [4] have established several sufficient conditions for the oscillation of solution of the second order nonlinear neutral delay differential equations of the form

$$
\frac{d}{d t}\left[r(t) \frac{d}{d t}[x(t)+p(t) x(\tau(t))]\right]+q(t) f(x(\sigma(t)))=0
$$

and

$$
\frac{d}{d t}\left[r(t)\left[\frac{d}{d t}[x(t)+p(t) x(\tau(t))]\right]^{\gamma}\right]+q(t) x^{\beta}(\sigma(t))=0,
$$

where $r, q, \tau, \sigma \in C\left(\mathbb{R}_{+}, \mathbb{R}_{+}\right), p \in C\left(\mathbb{R}_{+}, \mathbb{R}\right)$ and $\gamma, \beta$ are quotient of odd positive integers. Motivated by the above work, an attempt is made to study oscillatory behaviour of Equation (1) for $|p(t)|<+\infty$. Here we are connected with both $\left(A_{3}\right)$ and $\left(A_{4}\right)$.

Neutral functional differential equations have numerous applications in several field of the science as, for example, models of population growth and theory of population dynamics, fractal theory, nonlinear oscillation of earthquake, diffusion in porous media, fractional biological neurons, traffic flow, polymer theology, neural network modeling, fluid dynamics, viscoelastic panel in super sonic gas flow, real system characterized by power laws, electrodynamics of complex medium, sandwich system identification, nuclear reactors mathematical modeling of the diffusion of discrete particles in a turbulent fluid (see $[5-7,9]$ and the references cited therein). In last decades several results have been obtained on oscillation of nonneutral differential equations and neutral functional differential equations (see [10-15] and the references cited therein).

By a solution to Equation (1), we mean a function $x \in C\left(\left[T_{x}, \infty\right), \mathbb{R}\right), T_{x} \geq t_{0}$, which has the property $r z^{\prime} \in C^{1}\left(\left[T_{x}, \infty\right), \mathbb{R}\right)$ and satisfies Equation (1) on the interval $\left[T_{x}, \infty\right)$. We consider only those solutions to Equation (1) which satisfy condition $\sup \{|x(t)|: t \geq T\}>0$ for all $T \geq T_{x}$ and assume that Equation (1) possesses such solutions. A solution of Equation (1) is called oscillatory if it has arbitrarily large zeros on $\left[T_{x}, \infty\right)$; otherwise, it is said to be nonoscillatory. Equation (1) itself is said to be oscillatory if all of its solutions are oscillatory.

\section{Sufficient Conditions for Oscillation}

In this section, sufficient conditions are obtained for oscillatory and asymptotic behaviour of second order nonlinear neutral differential equations of the form (1).

Theorem 1. Let $0 \leq p(t) \leq p<1, t \in \mathbb{R}_{+}$. Assume that $\left(A_{1}\right)-\left(A_{3}\right)$ hold. Furthermore assume that

$\left(A_{5}\right) G$ and $H$ are nondecreasing and odd function

and

$\left(A_{6}\right) \int_{T}^{\infty}[q(\eta)+L v(\eta)] d \eta=\infty, L=\frac{H(\varepsilon)}{G(\varepsilon)}>0$ for $\varepsilon, T>0$

hold. Then every solution of the equation (1) is oscillatory.

Proof. Suppose for contrary that $x(t)$ is a nonoscillatory solution of equation (1). Then there exists $t_{0} \geq \rho$ such that $x(t)>0$ or $<0$ for $t \geq t_{0}$. Assume that $x(t)>0, x(t-\tau)>0$ and $x(t-\sigma)>0$ for $t \geq t_{0}$. From Equation (1), it follows that

$$
\left[r(t) z^{\prime}(t)\right]^{\prime}=-q(t) G\left(x\left(t-\sigma_{1}\right)\right)-v(t) H\left(x\left(t-\sigma_{2}\right)\right)<0,
$$

hold for $t \geq t_{1}>t_{0}$. Consequently, $r(t) z^{\prime}(t)$ is nonincreasing and $z^{\prime}(t), z(t)$ are of constant sign on $\left[t_{2}, \infty\right)$ for $t_{2}>t_{1}$. Let $r(t) z^{\prime}(t)<0$ for $t \geq t_{2}$. Then we can find $\varepsilon_{1}>0$ and a $t_{3}>t_{2}$ such that $r(t) z^{\prime}(t) \leq-\varepsilon_{1}$ for $t \geq t_{3}$. Integrating the relation $z^{\prime}(t) \leq-\frac{\varepsilon_{1}}{r(t)}$ from $t_{3}$ to $t\left(>t_{3}\right)$ and obtain $z(t) \leq z\left(t_{3}\right)-\varepsilon_{1}\left[\int_{t_{3}}^{t} \frac{d \eta}{r(\eta)}\right] \rightarrow-\infty$ as $t \rightarrow \infty$, a contradiction to the fact that $z(t)>0$ for $t \geq t_{1}$. Hence, $r(t) z^{\prime}(t)>0$ for $t \geq t_{2}$. As a result, $z(t)$ is 
nondecreasing on $\left[t_{2}, \infty\right)$. So, there exists $\varepsilon_{2}>0$ and a $t_{3}>t_{2}$ such that $z(t) \geq \varepsilon_{2}$ for $t \geq t_{3}$. On the other hand, $z(t)$ is nondecreasing implies that

$$
\begin{aligned}
(1-p(t)) z(t) & \leq z(t)-p(t) z(t-\tau) \\
& =x(t)+p(t) x(t-\tau)-p(t) x(t-\tau)-p(t) p(t-\tau) x(t-2 \tau) \\
& =x(t)-p(t) p(t-\tau) x(t-2 \tau) \leq x(t),
\end{aligned}
$$

that is, $(1-p) \varepsilon_{2} \leq x(t)$. Consequently, $x(t) \geq \varepsilon$ where $(1-p) \varepsilon_{2}=\varepsilon>0$. Therefore, (5) can be written as

$$
\left(r(t) z^{\prime}(t)\right)^{\prime}+G(\varepsilon)[q(t)+L v(t)] \leq 0 .
$$

We note that $\lim _{t \rightarrow \infty} r(t) z^{\prime}(t)$ exists. Integrating the last inequality from $t_{3}$ to $t\left(>t_{3}\right)$, then

$$
G(\varepsilon) \int_{t_{3}}^{t}[q(\eta)+L v(\eta)] d \eta \leq-\left[r(\eta) z^{\prime}(\eta)\right]_{t_{3}}^{t}<\infty, \text { as } t \rightarrow \infty,
$$

a contradiction due to the assumption $\left(A_{6}\right)$.

If $x(t)<0$ for $t \geq t_{0}$, then we set $y(t)=-x(t)$ for $t \geq t_{0}$ in (1) and using $\left(A_{5}\right)$ we find

$$
\left(r(t)(y(t)+p(t) y(t-\tau))^{\prime}\right)^{\prime}+q(t) G\left(y\left(t-\sigma_{1}\right)\right)+v(t) H\left(y\left(t-\sigma_{2}\right)\right)=0,
$$

then proceeding as above, we find a same contradiction. This completes the proof of the theorem.

Theorem 2. Let $1 \leq p(t) \leq p<\infty, t \in \mathbb{R}_{+}$and $G(p) \geq H(p)$. Assume that $\left(A_{1}\right)-\left(A_{3}\right)$ and $\left(A_{5}\right)$ hold. Furthermore assume that there exists $\lambda, \mu>0$ such that

$\left(A_{7}\right) G(u)+G(s) \geq \lambda G(u+s), H(u)+H(s) \geq \mu H(u+s)$ for $u, s \in \mathbb{R}_{+}$,

$\left(A_{8}\right) G(u s) \leq G(u) G(s), H(u s) \leq H(u) H(s)$ for $u, s \in \mathbb{R}_{+}$

and

$\left(A_{9}\right) \int_{T}^{\infty}\left[Q(\eta)+L_{1} V(\eta)\right] d \eta=\infty, L_{1}=\frac{\mu H(\varepsilon)}{\lambda G(\varepsilon)}>0$ for $T, \varepsilon>0$

hold, where $Q(t)=\min \{q(t), q(t-\tau)\}, V(t)=\min \{v(t), v(t-\tau)\}$. Then conclusion of the Theorem 1 is true.

Proof. Let $x(t)$ be a nonoscillatory solution of Equation (1). Proceeding as in Theorem 1, we have two cases: $r(t) z^{\prime}(t)<0$ and $r(t) z^{\prime}(t)>0$ for $t \in\left[t_{2}, \infty\right)$. The former case follows from Theorem 1 . Let's consider the later case. As a result, $z(t)$ is nondecreasing on $\left[t_{2}, \infty\right)$. So, there exists $\varepsilon>0$ and a $t_{3}>t_{2}$ such that $z(t) \geq \varepsilon$ for $t \geq t_{3}$. We note that $\lim _{t \rightarrow \infty} r(t) z^{\prime}(t)$ exists. From Equation (1), it is easy to see that

$$
\begin{aligned}
0= & \left(r(t) z^{\prime}(t)\right)^{\prime}+q(t) G\left(x\left(t-\sigma_{1}\right)\right)+v(t) H\left(x\left(t-\sigma_{2}\right)\right)+G(p)\left[\left(r(t-\tau) z^{\prime}(t-\tau)\right)^{\prime}\right. \\
& \left.+q(t-\tau) G\left(x\left(t-\tau-\sigma_{i}\right)\right)+v(t-\tau) H\left(x\left(t-\tau-\sigma_{2}\right)\right)\right]
\end{aligned}
$$

in which we use $\left(A_{7}\right),\left(A_{8}\right)$ and $z(t) \leq x(t)+p x(t-\tau)$ to obtain

$$
\begin{aligned}
0 & \left(r(t) z^{\prime}(t)\right)^{\prime}+G(p)\left(r(t-\tau) z^{\prime}(t-\tau)\right)^{\prime}+Q(t)\left[G\left(x\left(t-\sigma_{1}\right)\right)+G\left(p x\left(t-\tau-\sigma_{1}\right)\right)\right] \\
& +v(t) H\left(x\left(t-\sigma_{2}\right)\right)+G(p) v(t-\tau) H\left(x\left(t-\tau-\sigma_{2}\right)\right) \\
\geq & \left(r(t) z^{\prime}(t)\right)^{\prime}+G(p)\left(r(t-\tau) z^{\prime}(t-\tau)\right)^{\prime}+\lambda Q(t) G\left[x\left(t-\sigma_{1}\right)+p x\left(t-\tau-\sigma_{1}\right)\right] \\
& +v(t) H\left(x\left(t-\sigma_{2}\right)\right)+G(p) v(t-\tau) H\left(x\left(t-\tau-\sigma_{2}\right)\right) \\
\geq & \left(r(t) z^{\prime}(t)\right)^{\prime}+G(p)\left(r(t-\tau) z^{\prime}(t-\tau)\right)^{\prime}+\lambda Q(t) G\left(z\left(t-\sigma_{1}\right)\right)+v(t) H\left(x\left(t-\sigma_{2}\right)\right) \\
& +H(p) v(t-\tau) H\left(x\left(t-\tau-\sigma_{2}\right)\right)
\end{aligned}
$$

that is,

$$
\left(r(t) z^{\prime}(t)\right)^{\prime}+G(p)\left(r(t-\tau) z^{\prime}(t-\tau)\right)^{\prime}+\lambda Q(t) G\left(z\left(t-\sigma_{1}\right)\right)+\mu V(t) H\left(z\left(t-\sigma_{2}\right)\right) \leq 0
$$

for $t \geq t_{3}>t_{2}$. Consequently,

$$
\left(r(t) z^{\prime}(t)\right)^{\prime}+G(p)\left(r(t-\tau) z^{\prime}(t-\tau)\right)^{\prime}+\lambda Q(t) G(\varepsilon)+\mu V(t) H(\varepsilon) \leq 0 .
$$


Integrating the last inequality from $t_{3}$ to $t\left(>t_{3}\right)$, then

$$
\lambda G(\varepsilon) \int_{t_{3}}^{t}\left[Q(\eta)+L_{1} V(\eta)\right] d \eta \leq-\left[r(\eta) z^{\prime}(\eta)\right]_{t_{3}}^{t}+G(p)\left[r(\eta-\tau) z^{\prime}(\eta-\tau)\right]_{t_{3}}^{t}<\infty \text {, as } t \rightarrow \infty,
$$

a contradiction due to the assumption $\left(A_{9}\right)$. The case $x(t)<0$ is similar. Thus the theorem is proved.

Theorem 3. Let $-1 \leq p(t) \leq 0, t \in \mathbb{R}_{+}$. If $\left(A_{1}\right)-\left(A_{3}\right),\left(A_{5}\right)$ and $\left(A_{6}\right)$ hold, then every unbounded solution of Equation (1) oscillates.

Proof. Let on the contrary that $x(t)$ be a unbounded solution of Equation (1) on $\left[t_{0}, \infty\right), t_{0}>\rho$. Proceeding as in Theorem 1 , it concludes that $r(t) z^{\prime}(t)$ is nonincreasing and $z(t), z^{\prime}(t)$ are monotonicon $\left[t_{2}, \infty\right)$. Indeed, $z(t)<0$ for $t \geq t_{3}$ implies that $x(t) \leq x(t-\tau)$, and hence

$$
x(t) \leq x(t-\tau) \leq x(t-2 \tau) \leq \ldots \leq x\left(t_{3}\right)
$$

that is, $x(t)$ is bounded, which is absurd. Hence, $z(t)>0$ for $t \geq t_{3}$. Suppose that $r(t) z^{\prime}(t)>0$ for $t \geq t_{3}$. Clearly, $z(t) \leq x(t)$ implies that

$$
\left(r(t) z^{\prime}(t)\right)^{\prime}+q(t) G\left(z\left(t-\sigma_{1}\right)\right)+v(t) H\left(z\left(t-\sigma_{2}\right)\right) \leq 0
$$

for $t \geq t_{3}$. On the other hand, $z(t)$ is nondecreasing implies that, there exist $\varepsilon>0$ and a $t_{4}>t_{3}$ such that $z(t) \geq \varepsilon$ for $t \geq t_{4}$. Consequently, for $t_{5}>t_{4}+\sigma$, it follows from Equation (7) that

$$
\left(r(t) z^{\prime}(t)\right)^{\prime}+G(\varepsilon) q(t)+H(\varepsilon) v(t) \leq 0, t \geq t_{5}
$$

Integrating the last inequality from $t_{5}$ to $t\left(>t_{5}\right)$, we have

$$
G(\varepsilon) \int_{t_{5}}^{t}[q(\eta)+L v(\eta)] d \eta \leq-\left[r(s) z^{\prime}(s)\right]_{t_{5}}^{t}<\infty, \text { as } t \rightarrow \infty,
$$

a contradiction to $\left(A_{6}\right)$. Hence, $r(t) z^{\prime}(t)<0$ for $t \geq t_{3}$. Rest of the theorem follows from Theorem 1 . Thus, the proof of the theorem is complete.

Theorem 4. Let $-1<-p \leq p(t) \leq 0, t \in \mathbb{R}_{+}$and $p>0$. If all the assumptions of Theorem 3 hold, then every solution of Equation (1) either oscillates or converges to zero as $t \rightarrow \infty$.

Proof. Proceeding as in the proof of Theorem 1, we have obtained Equation (5) and hence $r(t) z^{\prime}(t)$ is nonincreasing on $\left[t_{2}, \infty\right)$. Therefore, $z(t)$ is monotonic on $\left[t_{3}, \infty\right), t_{3}>t_{2}$. So we have four cases namely:

1. $z(t)>0, \quad r(t) z^{\prime}(t)>0$,

2. $z(t)>0, \quad r(t) z^{\prime}(t)<0$,

3. $z(t)<0, \quad r(t) z^{\prime}(t)>0$,

4. $z(t)<0, \quad r(t) z^{\prime}(t)<0$.

Using the arguments as in the proof of Theorems 1 and Theorem 3, we get contradictions to $\left(A_{3}\right)$ and $\left(A_{6}\right)$ when the Case (2) and Case (1) respectively. Since $z(t)<0$ implies that $x(t)$ is bounded, that is, $z(t)$ is bounded, then the Case (4) is not possible due to Theorem $1\left(\because z^{\prime}(t)<0\right.$ implies that $\left.\lim _{t \rightarrow \infty} z(t)=-\infty\right)$.

Consequently, the Case (3) holds for $t \geq t_{3}$. In this case, $\lim _{t \rightarrow \infty} z(t)$ exits. As a result,

$$
\begin{aligned}
& 0 \geq \lim _{t \rightarrow \infty} z(t)=\limsup _{t \rightarrow \infty} z(t)=\limsup _{t \rightarrow \infty}(x(t)+p(t) x(t-\tau)) \\
& \geq \limsup _{t \rightarrow \infty}(x(t)-p x(t-\tau)) \\
& \geq \limsup _{t \rightarrow \infty} x(t)+\liminf _{t \rightarrow \infty}(-p x(t-\tau))=(1-p) \limsup _{t \rightarrow \infty} x(t)
\end{aligned}
$$

implies that $\lim \sup _{t \rightarrow \infty} x(t)=0[\because 1-p>0]$ and hence $\liminf _{t \rightarrow \infty} x(t)=0$. Thus $\lim _{t \rightarrow \infty} x(t)=0$. The case $x(t)<0$ is similar dealt with. This completes the proof of the theorem. 
Theorem 5. Let $-\infty<-p_{1} \leq p(t) \leq-p_{2}<-1, p_{1}, p_{2}>0$ and $t \in \mathbb{R}_{+}$. Assume that $\left(A_{1}\right)-\left(A_{3}\right),\left(A_{5}\right)$ and $\left(A_{6}\right)$ hold. If

$\left(A_{10}\right) \int_{T}^{\infty}\left[q(\eta)+L_{2} v(\eta)\right] d \eta=\infty, L_{2}=\frac{H\left(-p_{1}^{-1} \alpha\right)}{G\left(-p_{1}^{-1} \alpha\right)}>0$ for $T, p_{1}>0$ and $\alpha<0$,

then every bounded solution of Equation (1) either oscillates or converges to zero as $t \rightarrow \infty$.

Proof. Suppose on the contrary that $x(t)$ is a solution of Equation (1) which is bounded on $\left[t_{0}, \infty\right), t_{0}>\rho$. Using the same type of reasoning as in Theorem 1 , we have that $z^{\prime}(t)$ and $z(t)$ are of one sign on $\left[t_{2}, \infty\right)$ and have four possible cases like as in Theorem 4. Case (2) and Case (4) are not possible because of $\left(A_{3}\right)$ and bounded $z(t)$. Case (1) follows from the proof of the Theorem 3. For the Case (3), we claim that $\lim _{t \rightarrow \infty} z(t)=0$. If not, there exists $\alpha<0$ and $t_{3}>t_{2}$ such that $z\left(t+\tau-\sigma_{1}\right)<\alpha$ and $z\left(t+\tau-\sigma_{2}\right)<\alpha$ for $t \geq t_{3}$. Hence, $z(t) \geq p(t) x(t-\tau) \geq-p_{1} x(t-\tau)$ implies that $x\left(t-\sigma_{1}\right) \geq-p_{1}^{-1} \alpha>0$ and $x\left(t-\sigma_{2}\right) \geq-p_{1}^{-1} \alpha>0$ for $t \geq t_{3}$. Consequently, Equation (5) becomes

$$
\left(r(t) z^{\prime}(t)\right)^{\prime}+G\left(-p_{1}^{-1} \alpha\right) q(t)+H\left(-p_{1}^{-1} \alpha\right) v(t) \leq 0
$$

for $t \geq t_{3}$. Integrating the last inequality from $t_{3}$ to $t\left(>t_{3}\right)$, we get

$$
G\left(-p_{1}^{-1} \alpha\right) \int_{t_{3}}^{t}\left[q(\eta)+L_{2} v(\eta)\right] d \eta \leq-\left[r(s) z^{\prime}(s)\right]_{t_{3}}^{t}<\infty, \text { as } t \rightarrow \infty,
$$

a contradiction to $\left(A_{10}\right)$. Ultimately, $\lim _{t \rightarrow \infty} z(t)=0$. Hence,

$$
\begin{aligned}
0 & =\lim _{t \rightarrow \infty} z(t)=\liminf _{t \rightarrow \infty} z(t) \\
& \leq \liminf _{t \rightarrow \infty}\left(x(t)-p_{2} x(t-\tau)\right) \\
& \leq \limsup _{t \rightarrow \infty} x(t)+\liminf _{t \rightarrow \infty}\left(-p_{2} x(t-\tau)\right) \\
& =\left(1-p_{2}\right) \limsup _{t \rightarrow \infty} x(t)
\end{aligned}
$$

implies that $\limsup _{t \rightarrow \infty} x(t)=0\left[\because 1-p_{2}<0\right]$. Thus, $\liminf _{t \rightarrow \infty} x(t)=0$ and hence $\lim _{t \rightarrow \infty} x(t)=0$. Therefore, any solution $x(t)$ of Equation (1) converges to zero. The case $x(t)<0$ is similar. This completes the proof of the theorem.

Remark 1. If we denote $R(t)=\int_{t}^{\infty} \frac{d \eta}{r(\eta)}$, then $\left(A_{4}\right)$ implies that $R(t) \rightarrow 0$ as $t \rightarrow \infty$, since $R(t)$ is nonincreasing.

Theorem 6. Let $0 \leq p(t) \leq p<\infty, t \in \mathbb{R}_{+}$and $G(p) \geq H(p)$. Assume that $\left(A_{1}\right),\left(A_{2}\right),\left(A_{4}\right),\left(A_{5}\right)$ and $\left(A_{7}\right)-\left(A_{9}\right)$ hold. If

$\left(A_{11}\right) \int_{T}^{\infty} \frac{1}{r(\eta)}\left[\int_{T_{1}}^{\eta}\left\{Q(\zeta) G\left(\varepsilon R\left(\zeta-\sigma_{1}\right)\right)+L_{3} V(\zeta) H\left(\varepsilon R\left(\zeta-\sigma_{2}\right)\right)\right\} d \zeta\right] d \eta=\infty$ for $T, T_{1}, C>0$,

where $L_{3}=\frac{\mu}{\lambda}>0$ then also conclusion of the Theorem 1 is true, where $Q(t)$ and $V(t)$ is defined in Theorem 2.

Proof. On the contrary, we proceed as in Theorem 1 to obtain Equation (5) for $t \geq t_{1}$ and $r(t) z^{\prime}(t)$ is non increasing on $\left[t_{2}, \infty\right), t_{2}>t_{1}$. The case $r(t) z^{\prime}(t)>0$ for $t \geq t_{0}$ is same as in Theorem 2 and gives a contradiction due to $\left(A_{9}\right)$. Let's suppose that $r(t) z^{\prime}(t)<0$, for $t \geq t_{2}$. Therefore, for $s \geq t>t_{2}, r(s) z^{\prime}(s) \leq r(t) z^{\prime}(t)$ implies that

$$
z^{\prime}(s) \leq \frac{r(t) z^{\prime}(t)}{r(s)}
$$

Consequently,

$$
z(s) \leq z(t)+r(t) z^{\prime}(t) \int_{t}^{s} \frac{d \theta}{r(\theta)}
$$


Because of $r(t) z^{\prime}(t)$ is nonincreasing, we can find a constant $\varepsilon>0$ such that $r(t) z^{\prime}(t) \leq-\varepsilon$ for $t \geq t_{2}$. As a result, $z(s) \leq z(t)-\varepsilon \int_{t}^{s} \frac{d \eta}{r(\eta)}$ and hence $0 \leq z(t)-\varepsilon R(t)$ for $t \geq t_{2}$. Using the above fact in Equation (6), we get

$$
\left(r(t) z^{\prime}(t)\right)^{\prime}+G(p)\left(r(t-\tau) z^{\prime}(t-\tau)\right)^{\prime}+\lambda Q(t) G\left(\varepsilon R\left(t-\sigma_{1}\right)\right)+\mu V(t) H\left(\varepsilon R\left(t-\sigma_{2}\right)\right) \leq 0
$$

for $t \geq t_{3}>t_{2}$. Integrating the last inequality from $t_{3}$ to $t\left(>t_{3}\right)$, we obtain

$$
\left[r(\eta) z^{\prime}(\eta)\right]_{t_{3}}^{t}+G(p)\left[r(\eta-\tau) z^{\prime}(\eta-\tau)\right]_{t_{3}}^{t}+\lambda \int_{t_{3}}^{t}\left[Q(\eta) G\left(\varepsilon R\left(\eta-\sigma_{1}\right)\right)+L_{3} V(\eta) H\left(\varepsilon R\left(\eta-\sigma_{2}\right)\right)\right] d \eta \leq 0,
$$

that is,

$$
\begin{aligned}
\lambda \int_{t_{3}}^{t}\left[Q(\eta) G\left(\varepsilon R\left(\eta-\sigma_{1}\right)\right)+L_{3} V(\eta) H\left(\varepsilon R\left(\eta-\sigma_{2}\right)\right)\right] d \eta & \leq-\left[r(\eta) z^{\prime}(\eta)+G(p)\left(r(\eta-\tau) z^{\prime}(\eta-\tau)\right)\right]_{t_{3}}^{t} \\
& \leq-\left[r(t) z^{\prime}(t)+G(p)\left(r(t-\tau) z^{\prime}(t-\tau)\right)\right] \\
& \leq-(1+G(p)) r(t) z^{\prime}(t)
\end{aligned}
$$

implies that

$$
\frac{\lambda}{1+G(p)} \frac{1}{r(t)} \int_{t_{3}}^{t}\left[Q(\eta) G\left(\varepsilon R\left(\eta-\sigma_{1}\right)\right)+L_{3} V(\eta) H\left(\varepsilon R\left(\eta-\sigma_{2}\right)\right)\right] d \eta \leq-z^{\prime}(t) .
$$

Again integrating the last inequality, we obtain that

$$
\frac{\lambda}{1+G(p)} \int_{t_{3}}^{t} \frac{1}{r(\eta)}\left[\int_{t_{3}}^{\eta}\left\{Q(\zeta) G\left(\varepsilon R\left(\zeta-\sigma_{1}\right)\right)+L_{3} V(\zeta) H\left(R\left(\zeta-\sigma_{2}\right)\right)\right\} d \zeta\right] d \eta \leq-[z(\eta)]_{t_{3}}^{t} .
$$

Since $z(t)$ is bounded and monotonic, then it follows that

$$
\int_{t_{3}}^{t} \frac{1}{r(\eta)}\left[\int_{t_{3}}^{\eta}\left\{Q(\zeta) G\left(\varepsilon R\left(\zeta-\sigma_{1}\right)\right)+L_{3} V(\zeta) H\left(\varepsilon R\left(\zeta-\sigma_{2}\right)\right)\right\} d \zeta\right] d \eta<\infty,
$$

a contradiction to $\left(A_{11}\right)$. The case $x(t)<0$ is similar dealt with. This completes the proof of the theorem.

Theorem 7. Let $-1 \leq p(t) \leq 0, t \in \mathbb{R}_{+}$. Assume that $\left(A_{1}\right),\left(A_{2}\right)$ and $\left(A_{4}\right)-\left(A_{6}\right)$ hold. Furthermore assume that

$\left(A_{12}\right) \int_{T}^{\infty} \frac{1}{r(\eta)}\left[\int_{T_{1}}^{\eta}\left\{q(\zeta) G\left(\varepsilon R\left(\zeta-\sigma_{1}\right)\right)+v(\zeta) H\left(\varepsilon R\left(\zeta-\sigma_{2}\right)\right)\right\} d \zeta\right] d \eta=\infty$ for $T, T_{1}, C>0$

hold. Then conclusion of the Theorem 3 is true.

Proof. The proof of the theorem follows from the proof of the Theorems 3 and 6 and hence the details are omitted.

Theorem 8. Let $-1<-p \leq p(t) \leq 0, t \in \mathbb{R}_{+}$and $p>0$. If all the conditions of Theorem 7 are satisfied, then conclusion of the Theorem 4 is true.

Proof. The proof of the theorem follows from the proof of Theorems 4 and 7. Hence, the proof of the theorem is complete.

Theorem 9. Let $-\infty<-p_{1} \leq p(t) \leq-p_{2}<-1, t \in \mathbb{R}_{+}$and $p_{1}, p_{2}>0$. Assume that $\left(A_{1}\right),\left(A_{2}\right),\left(A_{4}\right)-\left(A_{6}\right)$, $\left(A_{10}\right)$ and $\left(A_{12}\right)$ hold. If

$\left(A_{13}\right) \int_{T}^{\infty} \frac{1}{r(\eta)}\left[\int_{T_{1}}^{\eta}\left\{q(\zeta)+L_{2} v(\zeta)\right\} d \zeta\right] d \eta=\infty$ for $T, T_{1}>0$,

where $L_{2}$ is defined in Theorem 5 , then conclusion of the Theorem 5 is true.

Proof. Proceeding as in the proof of the Theorem 5 we have four possible cases for $t \geq t_{2}$. First two cases are similar to the proof of Theorem 8. Case (3) is similar to the proof of Theorem 5. Hence, we consider the Case (4) only. Using the same type of reasoning as in the Case (3) of Theorem 8, we get Equation (8) and hence 


$$
H\left(-p_{1}^{-1} \alpha\right)\left[\int_{t_{3}}^{t}\left\{q(\eta)+L_{2} v(\eta)\right\} d \eta\right] \leq-r(t) z^{\prime}(t)
$$

Therefore,

$$
H\left(-p_{1}^{-1} \alpha\right) \int_{t_{3}}^{t} \frac{1}{r(\eta)}\left[\int_{t_{3}}^{\eta}\left\{q(\zeta)+L_{2} v(\zeta)\right\} d \zeta\right] d \eta \leq-[z(\eta)]_{t_{3}}^{t} \leq-z(t)<\infty, \text { as } u \rightarrow \infty,
$$

a contradiction to $\left(A_{13}\right)$. Rest of the theorem follows from the proof of the Theorem 5 . This completes the proof of the theorem.

\section{Final Comment and Examples}

In this section, we will be giving some simple remarks to conclude the paper.

Remark 2. In Theorem 1-Theorem 9, $G$ and $H$ is allowed to be linear, sublinear or superlinear. A prototype of the function $G$ and $H$ satisfying $\left(A_{2}\right),\left(A_{5}\right),\left(A_{7}\right)$ and $\left(A_{8}\right)$ is

$$
\left(1+\alpha|u|^{\beta}\right)|u|^{\gamma} \operatorname{sgn}(u) \quad \text { for } u \in \mathbb{R}
$$

where $\alpha \geq 1$ or $\alpha=0$ and $\beta, \gamma>0$ are reals. For verifying $\left(A_{6}\right)$, we may take help of the well-known inequality (see [16, p. 292])

$$
u^{p}+v^{p} \geq h(p)(u+v)^{p} \quad \text { for } u, v>0, \quad \text { where } \quad h(p):=\left\{\begin{array}{cc}
1, & 0 \leq p \leq 1, \\
\frac{1}{2^{p-1}}, & p \geq 1 .
\end{array}\right.
$$

We finalize the paper by presenting two examples, which show existence of main results.

Example 1. Consider the differential equation

$$
\frac{d}{d t}\left[e^{-4 t} \frac{d}{d t}[x(t)+x(t-\pi)]\right]+e^{t}\left(x\left(t-\frac{\pi}{2}\right)\right)^{3}+e^{t}\left(x\left(t-\frac{3 \pi}{2}\right)\right)^{3}=0 \quad \text { for } t \geq \pi
$$

where $r(t):=e^{-4 t}, p(t): \equiv 1, \tau:=\pi, q(t): \equiv e^{t}, \sigma_{1}:=\frac{\pi}{2}, G(u):=u^{3}, v(t):=e^{t}, \sigma_{2}=\frac{3 \pi}{2}$ and $H(u):=u^{3}$ for $t \geq \pi$ and $u \in \mathbb{R}$. All the assumptions of Theorem 1 can be verified. Hence, due to Theorem 1 , every solution of Equation (10) oscillates. Clearly $x(t)=\sin (t)$ for $t \geq \pi$ is a solution Equation (10).

Example 2. Consider the differential equation

$$
\frac{d}{d t}\left[\frac{1}{t^{2}} \frac{d}{d t}\left[x(t)-e^{-\pi} x(t-\pi)\right]\right]+4 \cosh (\pi) t\left[e^{-\frac{\pi}{2}}(t+1) x\left(t-\frac{\pi}{2}\right)+x(t-\pi)\right]=0 \quad \text { for } t \geq 2 \pi
$$

where $r(t):=\frac{1}{t^{2}}, R(t):=\frac{1}{t}, p(t): \equiv e^{-\pi}, \tau:=\pi, q(t):=4 e^{-\frac{\pi}{2}} \cosh (\pi) t(t+1), \sigma_{1}=\frac{\pi}{2}, G(u):=u, v(t):=$ $4 \cosh (\pi) t, \sigma_{2}:=\pi$ and $H(u):=u$ for $t \geq 2 \pi$ and $u \in \mathbb{R}$. All the assumptions of Theorem 7 can be verified. In particular, for $(A 11)$, we have

$$
\int_{2 \pi}^{\infty} \frac{1}{\eta} \int_{2 \pi}^{\eta} 4 \cosh (\pi) \zeta \frac{\varepsilon}{\zeta-\pi} d \zeta d \eta=\infty \text { for any } \varepsilon>0
$$

Hence, due to Theorem 7, every solution of Equation (11) oscillates, and such a solution is $x(t)=e^{t} \sin (t)$ for $t \geq 2 \pi$.

Acknowledgments: This work is supported by the Department of Science and Technology (DST), New Delhi, India, through the bank instruction order No. DST/INSPIRE Fellowship/2014/140, dated Sept. 15, 2014.

Author Contributions: All authors contributed equally to the writing of this paper. All authors read and approved the final manuscript. 
Conflicts of Interest: "The authors declare no conflict of interest."

\section{References}

[1] Baculíková, B., \& Džurina, J. (2011). Oscillation theorems for second order neutral differential equations. Computers $\mathcal{E}$ Mathematics with Applications, 61(1), 94-99.

[2] Li, T., Rogovchenko, Y. V., \& Zhang, C. (2013). Oscillation results for second-order nonlinear neutral differential equations. Advances in Difference Equations, 2013(1), 336.

[3] Santra, S. S. (2016). Existence of positive solution and new oscillation criteria for nonlinear first order neutral delay differential equations. Differential Equations \& Applications, 8(1), 33-51.

[4] Tripathy, A. K., Panda, B., \& Sethi, A. K. (2016). On oscillatory nonlinear second order neutral delay differential equations. Differential Equations \& Applications, 8, 247-258.

[5] Hale, J. K., \& Lunel, S. M. V. (2013). Introduction to functional differential equations (Vol. 99). Springer Science \& Business Media.

[6] Kilbas, A. A. A., Srivastava, H. M., \& Trujillo, J. J. (2006). Theory and applications of fractional differential equations (Vol. 204). Elsevier Science Limited.

[7] Miller, K. S., \& Ross, B. (1993). An introduction to the fractional calculus and fractional differential equations. John Wiley and Sons, Inc., New York.

[8] Podlubny, I. (1998). Fractional differential equations: an introduction to fractional derivatives, fractional differential equations, to methods of their solution and some of their applications (Vol. 198). Elsevier.

[9] Kilbas, A. A., Marichev, O. I., \& Samko, S. G. (1993). Fractional integral and derivatives (theory and applications). Gordon and Breach Science Publisher, Yverdon.

[10] Baculíková, B., Li, T., \& žurina, J. (2013). Oscillation theorems for second-order superlinear neutral differential equations. Mathematica Slovaca, 63(1), 123-134.

[11] Hasanbulli, M., \& Rogovchenko, Y. V. (2010). Oscillation criteria for second order nonlinear neutral differential equations. Applied Mathematics and Computation, 215(12), 4392-4399.

[12] Li, T., \& Rogovchenko, Y. V. (2014). Oscillation theorems for second-order nonlinear neutral delay differential equations,Abstract and Applied Analysis, 2014 (2014), Article ID 594190.

[13] Liu, Y., Zhang, J., \& Yan, J. (2015). Existence of oscillatory solutions of second order delay differential equations. Journal of Computational and Applied Mathematics, 277, 17-22.

[14] Tamilvanan, S., Thandapani, E., \& Dzurina, J. (2017). Oscillation of second order nonlinear differential equation with sublinear neutral term. Differential Equations \& Applications, 9(1), 29-35.

[15] Yan, J. (2011). Existence of oscillatory solutions of forced second order delay differential equations. Applied Mathematics Letters, 24(8), 1455-1460.

[16] Hildebrandt, T. H. (1963). Introduction to the Theory of Integration. New York-London: Academic Press.

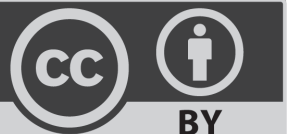

BY

(C) 2019 by the authors; licensee PSRP, Lahore, Pakistan. This article is an open access article distributed under the terms and conditions of the Creative Commons Attribution (CC-BY) license (http://creativecommons.org/licenses/by/4.0/). 